\title{
Periodontal disease is associated with coronary heart disease, preterm birth and low birth weight
}

\author{
Is periodontitis associated with an increased risk of coronary heart disease or \\ preterm and/or low birth weight births?
}

\begin{abstract}
Madianos PN, Bobetsis GA, Kinane DF. Is periodontitis associated with an increased risk of coronary heart disease and preterm and/or low birth weight births? J Clin Periodontol 2002; 29(Suppl. 3): S22-S36
\end{abstract}

Data sources MEDLINE and Embase were sources; reference lists from relevant articles, plus selected journals dated up to October 2001, were also searched by hand.

Study selection Studies were considered for inclusion if they addressed different aspects of periodontal disease (clinical, microbial or immunological) and clinical outcomes of coronary heart disease (CHD) or preterm and/or low birth weight (PLBW.) Because of the nature of the question, cross-sectional, case-control, cohort and clinical trial study-designs were considered.

Data extraction and synthesis Information regarding the quality and characteristics of studies was extracted independently by two reviewers using criteria relevant to each study design. Qualitative synthesis of the included studies was carried out due to extensive heterogeneity of the studies.

Results Half of the cohort studies (four out of eight), $75 \%$ of the case-control studies (three out of four) and half of the cross-sectional studies (two out of the four) reported a significant association between clinical measures of periodontitis and CHD (the increased risk ranged from 0 to 3.3 -fold). The periodontitis-PLBW association was evaluated in one cohort and two case-control studies. The cohort study as well as one of the two case-control studies reported a significant association between periodontitis and PLBW (odds ratios, 4.4-7.9).

Conclusions There is limited evidence that periodontitis is associated with an increased risk for CHD and PLBW. These associations should be explored further in well-designed observational and intervention studies to establish whether they are causal or incidental, to determine any possible benefits of intervention, and the potential to generalise.

\section{Commentary}

The aim of this systematic review was to evaluate existing evidence concerning the association between periodontal disease and (i) an increased risk for CHD and (ii) PLBW outcomes of pregnancy.

This is a relatively new field of investigation with comparatively few studies. Therefore, the goal was to determine whether there is an association and not a causal link. Because of this the authors elected to include cross-sectional studies, case-control studies, cohort studies and clinical trials, which have all provided varying levels of evidence that there is an association between the conditions. A determination of association, however, does not indicate a cause-effect relationship. Thus it would be premature to recommend periodontal therapy as an intervention for preventing CHD or PLBW.

It is also important to note that there were no published results of intervention studies or randomised controlled trials available for inclusion in the review, which would have provided the highest level of evidence for causation. The dilemma was whether to limit the review to only the highest study-quality standard (allowing no studies to be included in the review), or include studies with less than adequately defined interventions and outcomes (and reduce the quality of the evidence in the review).

The authors recognised the limitations in the studies available. They noted the heterogeneity of the studies as a major source of variability in the results and provided a thoughtful discussion of how specific differences in study designs may influence the study results. Given the paucity of well-designed studies in this area and the lack of clinical trial data, the authors justifiably conclude that there is a need for better evidence in this area. They suggest that clinicians await the results of larger prospective studies or of clinical trials before recommending clinical periodontal interventions to decrease risk for CHD or PLBW.

\section{Practice point}

- At this time there is no evidence to recommend clinical periodontal intervention to reduce CHD or PLBW.

\author{
Michelle M Henshaw \\ Massachusetts, USA \\ Evidence-Based Dentistry (2003) 4, 58. \\ doi:10.1038/sj.ebd.6400201
}

Department of Health Policy and Health Services Research, Boston University Goldman School of Dental Medicine, Boston, 